\title{
Research and Design on On-line Monitoring of Mechanical Properties of High Voltage Circuit Breaker
}

\author{
Jiajia Song \\ College of IOT Engineering. \\ Hohai University \\ Changzhou, China \\ e-mail: 457590744@qq.com \\ Jinbo Zhang \\ College of IOT Engineering \\ Hohai University \\ Changzhou, China \\ e-mail: zjb861@163.com
}

\author{
Bin Chen \\ College of IOT Engineering. \\ Hohai University \\ Changzhou, China \\ e-mail: 1003180410@qq.com
}

\author{
Zhengyong Xiong \\ Pacific AutomationTechnology co., LTD \\ Changzhou, China
}

\begin{abstract}
The statistics of International Meeting shows that most circuit breaker faults comes from mechanical side, so it is of great significance to do research on mechanical fault diagnosis. The implementation of circuit breaker mechanical properties on-line monitoring is an inevitable trend of the development of state maintenance mechanism, it is also an important part of intelligent circuit breaker research in the future. According to the requirement of the smart grid in national construction, it has more advanced demands to intelligent circuit breaker on-line monitoring. The demands not only include monitoring on contact temperature rise and current of the circuit breaker, but also include on-line monitoring of circuit breaker mechanical properties. So this paper designs an on-line monitoring device to measure the mechanical properties of circuit breaker. It is proved that it can meet the requirements of on-line monitoring of mechanical properties after practice.
\end{abstract}

Keywords- circuit breaker; on-line monitoring; coil current; temperature rise; travel curve

\section{INTRODUCTION}

In recent years, with the prosperity and development of economy, as well as the constantly increasing capacity and energy of the power system demand, there is higher request to the reliability and economy of power system. High voltage circuit breaker as the connection between the generation and distribution, its reliable operation has great significance to ensure the safety of the power grid. In the power system, high voltage circuit breaker has two aspects function: one is to control function, according to the power grid operation requirement, it can put part of the electrical equipment or lines into or out of running state, turn them to space or repair status. The other function is protection. When electrical equipment or circuits break down, high voltage circuit will be action under the protection of relay and automatic device and the fault section will be quickly removed from the power grid so that the power grid can operate normally and under fault-free.
International conference has had two world scope investigations on the reliability of high voltage circuit, at the same time, our country has also made a lot of statistical analysis of high voltage switch accidents. Corresponding investigation and statistics reports show that mechanical reasons account eighty percent for the malfunction of high voltage circuit breaker. Most of the fault is the problems of operating mechanism. The past inspection to the mechanical characteristic of high voltage circuit breaker is mainly in the time of equipment transfer and the power failure and in the maintenance periodic having a preventive test, such as replacing the components and checking the operating mechanism of the mechanical properties. Since the huge number of high voltage circuit breaker in the power system, the amount of maintenance is large and the cost is very high. According to the relevant statistical data, more than half substation maintenance costs is used in high voltage circuit breaker, and $60 \%$ of which is used on the minor repairs and routine maintenance of the circuit breaker. In addition, according to statistics, $10 \%$ of circuit breaker failure is caused by improper maintenance. The overhaul of circuit breaker lead the breaker completely collapse which wastes a lot of time and money. Disintegration and reassemble will cause many new defects, so how to effectively detect the work condition of the circuit breaker, discover the early failure of the breaker in time, treat the defective parts early, avoid vicious development of circuit breaker failure, prevent the occurrence of malignant accidents such as circuit breaker explosion is important for the safe and reliable operation of the power grid.

The status detection of circuit breaker creates the condition for the transition from plan maintenance to the state overhaul. For a long time, due to the disintegration of blind disassembly, the plan maintenance has wasted a great deal of manpower, material resources, financial resources and caused a power outage loss and reduced the service life of equipment. At present, each unit of the power 
system is committed to the transition of high voltage circuit breaker from plan maintenance to the state overhaul, no longer uses the number of year of the investment and the number of movements as standard, but uses the actual state of the equipment for the maintenance basis. In recent years, it has been found that relying on equipment on-line monitoring and diagnosis technology to realize the state of the equipment maintenance can achieve the following requirements of power system : (1) Due to the quality of goods, the reliability of equipment operation may be affected. Using the online monitoring can find the accidents in time under the equipment operation, nipping in the bud; (2) Using online monitoring to instead of a power outage test gradually, reducing the outage time of equipment, and cutting down the cost of test; (3) For the aging equipment or the equipment with known defects and hidden trouble, using online monitoring to monitor the running situation at any time so that the equipment will be quit once found out problems which can maximum use the residual life of equipment.

Because the existing high voltage circuit breaker monitoring device only can monitor the temperature rise of the contactor, lacking of mechanical features of online monitoring, lacking of adequate data accumulation and the analysis ability of mechanical fault diagnosis is insufficient, therefore, the research work of mechanical properties of high voltage circuit breaker monitoring technology has important academic significance and practical value.

\section{OPERATION STRUCTION AND MECHANICAL LIFE HIGH VOLTAGE CIRCUIT BREAKER}

Operating mechanism is an important component of the high voltage circuit breaker. The switching device with contactor can only open and close circuit through opening or closing the contactor, in consequence, this must rely on a certain mechanical operation system. The operation structure of circuit breaker consists of energy storage unit, control unit and power transfer unit.

The operation structure of high voltage circuit breaker has many forms, such as spring operating mechanism, pneumatic operation mechanism, hydraulic operating mechanism, hydraulic spring mechanism and so on. The contactor of high voltage circuit breaker can open and close reliably under various condition. It is mainly completed by the action of operation energy and transmission mechanism, the characteristic of its action is that the agency system is in an instantaneous change process when performing a task and completing the task, so the agency action has shock, vibration and other transient properties; in the closing current position, it may not act for a long time, but once there is an accident, it is required to act accurately and reliably. Because of the characteristic of the circuit breaker on agency action, the demand for the reliability of high voltage circuit breaker operating mechanism and transmission mechanism is very important. According to relevant data statistics, 70\% $80 \%$ of high voltage circuit breaker operation accidents is caused by circuit breaker mechanical problems, the same as the circuit breaker electrical loss, when the circuit breaker cuts the short circuit current, the damage the parts also has a long-term accumulation process, after operation for many times, the strength and stiffness of some metal parts in transmission and operating mechanism will be worse, and corrosion will cause deformation, then the circuit breaker is difficult to disconnect. Although in the situation of breaking small current, the influence on contact electrical life is very small, even if it switches for many times, it still has not reached its life, however, every time for breaking and a certain action time and speed will produce a certain degree of damage to the mechanical parts of circuit breaker. So, another factor that must be taken into consideration is the mechanical life of circuit breaker [8 - 12].

As is known to all, compared with other electrical equipment, machinery parts of the breaker is much more, in addition, the action of these parts is frequent, so the possibility of failure is large. As can be seen from the statistical analysis of more than $6 \mathrm{kV}$ high voltage switch failure reasons by electric power research institute in our country, in refusing action and error action failure, operating mechanism accounts for $41.63 \%$. International Council on Large Electric systems (CIGEI) data also shows that the operating mechanism failure accounts for $43.5 \%$,thus, both at home and abroad, the main cause of mechanical failure is the circuit breaker failure, so the monitoring on circuit breaker machinery status and health diagnosis is very important.

\section{THE MECHANICAL PROPERTIES OF INTELLIGENT CIRCUIT BREAKER AND ITS ON- LINE MONITORING}

The on-line monitoring on the mechanical properties of intelligent circuit breaker mainly focuses on monitoring the current when the circuit breaker is closing and the closing time-travel together with the beginning separated and closed points, with these above-mentioned statistics we can judge circuit breaker performance. The devices that should be used in the on-line monitoring include: threephase pressure sensor components, three-phase position sensor components, coil current acquisition sensor components, signal conditioning circuit, microprocessor circuit, display circuit, communication interface circuit and the related embedded expert decision software. The threephase pressure sensor components mainly monitor the breaker points and just point. The three-phase position sensor components mainly monitor the mechanical properties of the circuit breaker. The coil current acquisition sensor components monitor the motor coil current inside the circuit breaker operating mechanism. The signal conditioning circuit pre-process the collected signal, eliminate disturbance signal so that the microprocessor can collect the signals easily. The display circuit is used to display the measuring result. The communication interface circuit communicate with background management system. The embedded expert decision software is mainly used to analysis the gathered statistics and judge the fault.

\section{A. On-line monitoring of the circuit breaker closing and break-brake coil current}

Usually the electromagnet is used as first level control of the high-voltage circuit-breaker, most of the current in the control power of the circuit-break is direct. As a result 
we can get the important information that helps diagnosis mechanical failure from the current waveform of DC electromagnetic coil. The inductance size(L) of the electric magnet is depend on the the size of the coil and iron yoke iron core and has a related relationship with the travel of the core(S)(the journey that the core moves upward) and the size increases with the increase of S. In this thesis we use a hall sensor in order to fulfil the monitoring of its electromagnetic coil current. The drive current of the electromagnetic coil is $1-2 \mathrm{~A}$, the coil current and time curves are shown in Fig. 1.

A- electromagnet connecting rod, spring deformation. $\mathrm{C} \backslash \mathrm{D}$ - connecting rod position will soon arrive in the deepest position. E-connecting rod reaches the deepest place. F- opening of the main contact. G-opening of the auxiliary contact. H-Electromagnet connecting rod restore the initial position, spring restore the original shape.

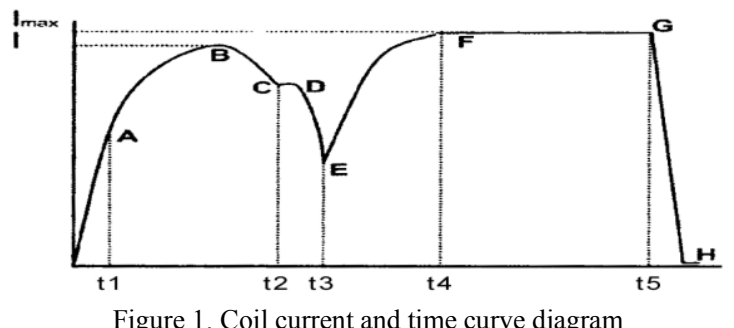

We can determine the basic mechanical situation of the circuit breaker through these curves. For example, if Point $\mathrm{B}$ rises, that means current increases in the movement process and we can conclude that circuit breaker may have jammed; if Point $\mathrm{G}$ moves to the right, that means the average speed of circuit breaker droops; if there is no curve that means the coil circuit breaker disconnects.

\section{B. On-line monitoring of the route, speed and mechanical life of the circuit breaker operating mechanism.}

When monitoring the route of the break we can use grating distance sensor and resistance sensor, when installed on the mechanism that moves straight we can use straight-line distance sensor, when installed on the rotating shaft of the operating mechanism we can use rotary electrical sensor. We can get the travel time curve related to the operation process of the circuit breaker, by a series of processing the output pulse signal of the sensor including photoelectric isolation, plastic, logical processing, data acquisition. With the help of the travel time curve we can calculate a series of parameters: average speed, average speed within $10 \mathrm{~ms}$ before or after closing electric circuit. One difficulty of the on-line monitoring is that the distance sensor cannot be installed on the moving contact head, so we can't measure the travel of the contact directly, thus we should convert. Fig .2 is the principle diagram of the circuit breaker mechanical properties testing. In the picture, the displacement sensor is fixed on the bottom of the circuit by using the stents, with the vacuum bubble moving up and down through connecting rod drives the sliding displacement sensor handle move up and down, translate the displacement signal into electrical signal and sent it to the monitoring instrument with the help of the amphenol connector.

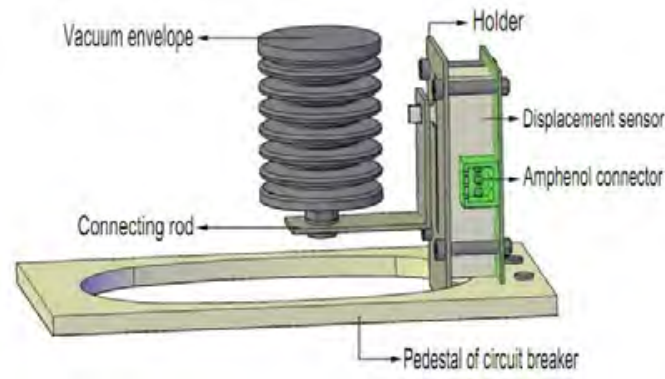

Figure 2. The principle diagram of the circuit breaker mechanical properties testing

We can not only monitor circuit breaker break-brake time - route curve, circuit breaker closing time - route curves, average circuit breaker brake speed, average circuit breaker closing speed, we can also monitor the mechanical life of the circuit breaker(number of times the vacuum circuit breaker actions ).

\section{Design of embedded software and XDSS (expert Decision Support System)}

All the embedded software will be researched and developed based on the latest ARM9 technology. We will make full use of the high speed computing and high extensibility of ARM9 and will implement the intelligent of the acquisition terminal. The electrical wear of the circuit breaker contact is one of the important factors affecting the electrical service life of circuit breaker. It can monitor the rest mechanical life of the circuit breaker online through statistics of all previous vacuum circuit breaker action. We can give the corresponding alarm information before breaker ending its life, so that we can ensure the safety of equipment operation. At the same time, the XDSS can detect the points closing time - waveform, current waveform of the energy storage motor, diagnose the state of the circuit breaker, alarm when the the abnormal situation occurs, give the solution to solve the problem. The black box which keeps the operation data of the full life circle of equipment will keep detailed records of equipment running status, for example: when and what was the operation, cumulative number of operations, etc, can facilitate the on-site maintenance personnel to check the equipment information and when the failure occurs it can help the maintenance personnel analysis reason and make responsibility identification.

\section{THE RESULTS}

When testing, the rated current of the circuit breaker is $1250 \mathrm{~A}$, the rated voltage is $10 \mathrm{Kv}$, and it is a vacuum circuit breaker. Operating mechanism is the spring one, three phase displacement sensor is chosen with sliding handle plastic potentiometer, and the total trip is $30 \mathrm{~mm}$, threephase pressure sensors are chosen $30 \mathrm{KN}$.

Designed by the above-mentioned principle, the mechanical characteristics of high voltage circuit breaker online monitoring device can monitor the break-brake coil current curve, the close-brake coil current curve, whether the break-brake coil has broken lines, whether the closebrake coil has broken lines, energy storage coil current curve, energy storage time, break-brake time - stroke curve, close-brake time - stroke curve, the average speed of circuit breaker braking, average speed of circuit breaker closing. Fig .3 shows the measured circuit breaker braking/closing time-stroke and current curve. 

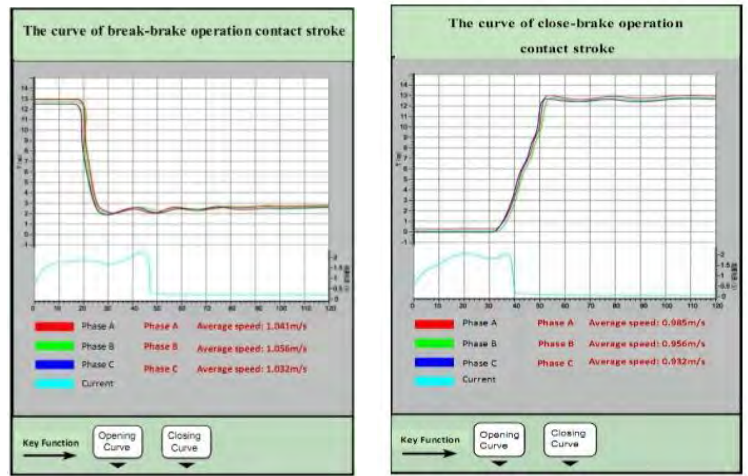

Figure 3 the circuit breaker mechanical properties braking/closing timestroke, current curve

TABLE I The measured value of average braking speed and average closing speed of the circuit breaker

\begin{tabular}{|c|c|c|c|}
\hline $\begin{array}{c}\text { Average braking } \\
\text { speed }\end{array}$ & $\begin{array}{c}\text { value } \\
(\mathrm{m} / \mathrm{s})\end{array}$ & $\begin{array}{c}\text { Average } \\
\text { closing } \\
\text { speed }\end{array}$ & $\begin{array}{c}\text { value } \\
(\mathrm{m} / \mathrm{s})\end{array}$ \\
\hline Phase A & 1.041 & phase A & 0.985 \\
\hline phase B & 1.056 & phase B & 0.956 \\
\hline phase C & 1.032 & phase C & 0.932 \\
\hline
\end{tabular}

Currently, offline mechanical properties tester measures the beginning closed points, beginning separated points through putting voltage excitation signal on the dynamic and static contact. It determines the beginning closed points or beginning separated points according to the presence of current responding signal generated by excitation, and then measures the spacing and overrun through the beginning closed and beginning separated points. Spacing is the distance from opening to closing moments; overrun is the stroke from the beginning closed points to the completely closing stable situation, that is the stroke from the moment of beginning closed points to the moment of completely closed (generally about $3 \mathrm{~mm}$ ).

For the on-line monitoring of circuit breaker mechanical properties, it is impossible to take this way. The main reason is that there is high voltage when the circuit breaker working, it can't put excitation signal, so it is difficult to judge the beginning closed points and the beginning separated points. So it can't realize the monitoring of spacing, overrun, braking and closing time, the different period of braking and closing, the measurement of these parameters all demand the beginning separated and closed points to make determination.

In order to make online monitoring of the circuit breaker spacing, overrun, the braking and closing time, the different period of braking and closing, it adopts the pressure sensors to obtain the beginning separated and closed points. The pressure sensors are installed under the rod of circuit breaker, judging the beginning separated or the beginning closed points according to the spring compression force (that is the presence of pressure).

For the actual operation of circuit breakers, the monitoring on its mechanical properties will mainly store the mechanical properties for the early coming into operation. Compared the historical curve with the curve putting into operation, if the envelope deviates by $\pm 5 \%$, we conclude that the circuit breaker mechanical structure has problems, and bring out maintenance or repair advice for it.

\section{CONCLUSIONS}

The on-line monitoring device on the mechanical properties of circuit breaker designed by the above-mentioned principle has been put into application in many domestic projects. It has become a trend of intelligent circuit breaker. The monitoring device can monitor the break-brake coil current curve, the close-brake coil current curve, whether the break-brake coil has broken lines, whether the close-brake coil has broken lines, energy storage coil current curve, energy storage time, breakbrake time - stroke curve, close-brake time - stroke curve; but as for spacing, overrun, the braking and closing time, the different period of braking and closing, these parameters need the beginning separated and closed points to measure. But the beginning separated and closed points require putting three sets of voltage excitation signal on the dynamic and static contact, and then judge the beginning separated and closed points according to the presence of excitation signal. For the on-line monitoring, it is impossible to put excitation signal in the high voltage circuit breaker, there we put forward the pressure sensor to judge the beginning separated and closed points, that is to judge the beginning separated and closed points by the presence of pressure. But the main problem of the method is that it can only apply to the new operation of circuit breaker, for these had been put into operation, it can't install.

\section{ACKNOWLEDGMENT}

This paper has obtained funding from Science and Technology Support Project in Jiangsu Province, and the item number is BE2014052.

\section{REFERENCES}

[1] Li Shen, Yulong Huang, Jiali Qian. The research on monitoring of high voltage circuit breaker [J]. Proceedings of the csee, 1997, (2) : 113-117

[2] Qingpeng Meng. The research on the detection of the high voltage circuit breaker mechanical state based on the analysis of vibration signal[D]. Xi 'an jiaotong university, 2006

[3] Yue'e Yao, Ting Zhang, Chun Liu,etc. The online information processing of circuit breaker mechanical state $[\mathrm{J}]$. High voltage electric equipment2004,40 (2) : 103-111K. Elissa, "Title of paper if known," unpublished.

[4] Lingjie Huang. The research on the inspection of high voltage circuit breaker status and fault diagnosis methods[D].Beijing jiaotong university, 2007

[5] Wu Yang, Xiaohua Wang, Mingzhe Rong, etc. The research on the on-line monitoring sensors of the high voltage electric equipment temperature based on infrared temperature measurement technology. Proceedings of the csee, 2002, 22(9) : 114-117

[6] Yong Zhang, Feng Chen. The statistical analysis of distribution operation accidents in Changsha region.

[7] Qigui Yu. Several important technical index in the electrical design $[\mathrm{J}]$. High voltage electrical technical information, 2011,7(6):9-11.

[8] Wenjing He, Yi Zhao. Electrical fiber Bragg grating temperature fire early warning technology $[\mathrm{J}]$. Fire science and technology, 2011,7(6):9-11.

[9] Sun Zhenglai. The research on the on-line monitoring of high voltage switch cabinet temperature. [J]. ELECTRIC POWER IT,2008,6(6):62-66.

[10] Thermo Physieaminima. Thermoelectric infrared sensors(thermopiles) for remote temperature measurements[R]. PerkinElmer Company,2000. 
[11] L.Balgard ,L.Lundin. Monitoring Primary Circuit Temperature and Breakers Condition in MV Substations[J]. ABB Review,1993, (3):21-26.

[12] The diagnosis of И В. Kong DE la ke. Now hydropower station equipment [J]. Water conservancy and hydropower letters,2003,24(19): 5-8 